\title{
ANALYSIS OF STABILITY PROBLEMS VIA MATRIX LYAPUNOV FUNCTIONS ${ }^{1}$
}

\author{
ANATOLY A. MARTYNYUK ${ }^{2}$ \\ Institute of Mechanics, the Ukranian Academy of Sciences, \\ Nesterov Str. 3, 252057 \\ Kiev-57, USSR
}

\begin{abstract}
The stability of nonlinear systems is analyzed by the direct Lyapunov's method in terms of Lyapunov matrix functions. The given paper surveys the main theorems on stability, asymptotic stability and nonstability. They are applied to systems of nonlinear equations, singularly-perturbed systems and hybrid systems. The results are demonstrated by an example of a twocomponent system.
\end{abstract}

Key words: Lyapunov Matrix Function, Stability, Asymptotic Stability, Nonlinear Systems.

AMS Subject Classification: 34D20, 34A34.

\section{INTRODUCTION}

In 1979, A. A. Martynyuk (see [32]) gave a description of two-indexed system of functions called matrix Lyapunov function (MLF). In later works, [17, 20, 22], some main concepts of MLF's were developed. As it is well-known in the theory of Lyapunov stability, the notion of definiteness of the scalar Lyapunov function $\mathrm{V}$ is of importance, for $\mathrm{V}$ is a positive measure in one of the variables. The definiteness remains essential also for MLF's.

\footnotetext{
${ }^{1}$ Received: April 1990, Revised: October 1990

${ }^{2}$ This work has been completed during the author's short visit at Florida Institute of Technology, U.S.A.
} 


\section{THE DEFINITENESS OF MATRIX-FUNCTIONS}

Let $\left(\mathbb{R}^{n},\|\cdot\|\right)$ be a real Euclidian normed space. Denote $B(\rho)=B(0, \rho)=\{x:\|x\|<\rho\}$ an open ball centered at the origin with radius $\rho$; let $D=\overline{\mathbb{R}} \times B(\rho)$ be the Cartesian product of $\overline{\mathbb{R}}=[-\infty,+\infty]$ and $B(\rho)$. Moreover, we shall consider an open connected time-invariant neighborhood $N \subseteq \mathbb{R}^{n}$ of point $x=0$ and intervals $T_{0}=\left\{t: t_{0} \leq t<+\infty\right\}, \mathrm{t}_{0} \in \mathrm{T}, \mathrm{T} \subseteq \mathbb{R}$ and $T_{\tau}=\{\mathrm{t}: \tau \leq \mathrm{t}<+\infty\}, \tau \in \mathbb{R}$, where $T$ is the set of initial values.

Consider a perturbed motion equation

$$
\frac{d x}{d t}=f(t, x), f(\mathrm{t}, 0)=0,
$$

where $x \in \mathbb{R}^{n}$ and $f \in C\left(D, \mathbb{R}^{n}\right)$.

Suppose that a solution $\chi\left(t ; t_{0}, x_{0}\right)$ of $(2.1)$ is continuous for all $t \in T_{0}$ and $\chi\left(t_{0} ; t_{0}, x_{0}\right)=x_{0}$.

Since the definitions of stability of the trivial solution of (2.1) with respect to $T$, attraction with respect to $T$ and asymptotic stability with respect to $T$ are given in $[5,7]$, and the same concepts relative to a part of variables are introduced in [16], we omit this notions here.

Matrix-Function (MF). Together with (2.1) we consider a two-indexed system of functions

$$
U(t, x)=\left(u_{i j}(t, x)\right), \quad i, j \in\{1, \ldots, m\}, m>1
$$

with $u_{i j} \in C\left(T_{\tau} \times B(\rho), \mathbb{R}\right)$.

The notion of the definiteness of an auxiliary function (that is used in the direct Lyapunov's method) is a main one [15], since this behaves as a scalar function having all norm properties. The definiteness of the MF can be defined in terms of the definiteness of a corresponding scalar function whose construction is based on the MF.

By means of the matrix-function $\mathrm{U}$, we can introduce the following scalar functions

$1^{0} . \quad U_{0}(t, x)=\max \left\{u_{i j}(t, x): 1 \leq i, j \leq m\right\}$;

$2^{0}$. $\quad U_{0}(t, x)=\phi^{\mathrm{T}} U(t, x) \phi$, where $\phi \in \mathbb{R}_{+}^{m}$ or $\phi \in \mathrm{C}\left[\mathbb{R}^{n}, \mathbb{R}_{+}^{m}\right], \phi(0)=0$;

$3^{0} . \quad U_{0}(t, x)=\sum_{i, j=1}^{m} u_{i j}(t, x)$.

Definition 2.1. Matrix-function $U: \mathrm{T}_{\tau} \times \mathbb{R}^{n} \rightarrow \mathbb{R}^{m \times m}$ is called

1) positive definite on $T_{\tau}$ iff there exists a time-invariant connected neighborhood $N \subseteq \mathbb{R}^{n}$ of point $x=0$, a positive definite function, $w: N \rightarrow \mathbb{R}_{+}$, and a vector $\phi \in \mathbb{R}_{+}^{m}$ or a vector-function $\phi \in C\left[\mathbb{R}^{n}, \mathbb{R}_{+}^{m}\right], \phi(0)=0$ such that

a) $\quad U=C\left(T_{\tau} \times N, \mathbb{R}^{m \times m}\right)$; 
b) $\quad U(t, 0)=0 \forall t \in T_{\tau}$;

c) function $U_{0}(t, x)$, defined by $1^{0}, 2^{0}$ and $3^{0}$, satisfies the condition

$$
U_{0}(t, x) \geq w(x) \quad \forall(t, x \neq 0) \in T_{\tau} \times \mathbb{R}^{n} .
$$

2) positive definite on $T_{\tau} \times S$ iff the above conditions a) - c) are satisfied for $N=S$ (where $S \subseteq \mathbb{R}^{n}$ is an arbitrary set);

3) positive definite in the whole on $T_{\tau}$ iff the conditions a) - c) are satisfied for $N=\mathbb{R}^{n}$;

4) negative definite (in the whole) on $T_{\tau} \times N$ (on $T_{\tau}$ ) iff $(-U)$ is positive definite (in the whole) on $T_{\tau} \times N$ (on $T_{\tau}$ ).

Remark 1. The expression "on $T_{\tau}$ " in Definition 2.1 will be dropped if all above requirements are satisfied for every $\tau \in \mathbb{R}$.

Remark 2. If the $(i, j)$-th element $u_{i j}: \mathbb{R}^{n} \rightarrow \mathbb{R}$ does not depend on $t \in T_{\tau}$, the conditions of Definition 2.1 are simplified, in particular, condition $1 \mathrm{c}$ ) is reduced to

$$
U_{0}(x)>0 \quad \forall(x \neq 0) \in \mathbb{R}^{n},(\phi \neq 0) \in \mathbb{R}^{m}\left(\mathbb{R}_{+}^{m}\right) .
$$

Remark 3. The definition of the semidefiniteness of the MF is formulated via Definition 2.1 and Definition 10 from [7, p. 23].

Remark 4. In the case when $m=1$ and $T_{\tau}=\mathbb{R}$, Definition 2.1 is reduced to the original definitions of positive semidefiniteness (in the whole) introduced earlier by Lyapunov [15] and Barbashin/Krassovsky [11].

Definition 2.2. Matrix-function $U: T_{\tau} \times \mathbb{R}^{n} \rightarrow \mathbb{R}^{m \times m}$ is called

1) decreasing on $T_{\tau}, \tau \in \mathbb{R}$, iff there exist a time-invariant neighborhood $N \subseteq \mathbb{R}^{n}$ of point $x=0$, a positive definite function $u: N \rightarrow \mathbb{R}$, and a vector $\phi \in \mathbb{R}_{+}^{m}$ such that the conditions $1 \mathrm{a}$ ) and $1 \mathrm{~b}$ ) of Definition 2.1 are satisfied and

$$
U_{0}(t, x) \leq u(x) \quad \forall(x \neq 0) \in \mathbb{R}^{n}, t \in T_{\tau}
$$

2) decreasing on $T_{\tau} \times S$, iff the above conditions 1) hold for $N=S\left(S \subseteq \mathbb{R}^{n}\right)$;

3) decreasing in the whole on $T_{\tau}$ if the conditions of 1) hold for $N=\mathbb{R}^{n}$.

\section{MATRIX LYAPUNOV FUNCTION}

First observe that the definiteness of MF's [17] was based on a generalization of the socalled "maximum function" introduced by LaSalle [14] for the case of vector functions. Later [18, 30], the concept of the definiteness has been modified, however no formal definition was introduced. We now introduce the definition of MLF's based on the definiteness of the MF from 
Section 2 (whose special case agrees with the classical definiteness via Lyapunov [15] (cf., $[11,12,38])$.

We define the total derivatives of the matrix-function (2.2) by virtue of system (2.1) as

$$
D^{+} U(t, x)=\left(D^{+} u_{i j}(t, x) ; i, j \in\{1, \ldots, m\}\right),
$$

where

$$
\begin{gathered}
D^{+} u_{i j}(t, x)=\lim \sup \left\{\left[u_{i j}(t+\theta, x+\theta f(t, x))-u_{i j}(t, x)\right] \theta^{-1}: \theta \rightarrow 0^{+}\right\} \\
D_{+} U(t, x)=\left(D_{+} u_{i j}(t, x) ; i, j \in\{1, \ldots, m\}\right)
\end{gathered}
$$

where

$$
D_{+} u_{i j}(t, x)=\lim \inf \left\{\left[u_{i j}(t+\theta, x+\theta f(t, x))-u_{i j}(t, x)\right] \theta^{-1}: \theta \rightarrow 0^{+}\right\} .
$$

Unless specified, $D^{*} U$ is either $D^{+} U(t, x)$ or $D_{+} U(t, x)$.

Definition 3.1. The two-indexed system of functions (2.2) is called a matrix Lyapunov function for system (2.1) if on $T_{\tau} \times N$

a) the function $U$ is definite;

b) $\quad D^{*} U$ is semidefinite and has either the same sign as $U$ or the opposite sign of $U$.

It is clear that the function $U$ (cf. (2.2)) and it's total derivative in terms of (2.1) solves the stability problem of state $x=0$ of equation (2.1).

Definition 3.1 can be specified for various dynamic properties of state $x=0$. Denote $S(\phi)$ the set of MLF's that solve the stability problem.

Definition 3.2. A MF $U: \quad T_{\tau} \times \mathbb{R}^{n} \rightarrow \mathbb{R}^{m \times m}$ is called a MLF of $S(\phi)$ - type on $T_{\tau} \times N$ if

1) $U$ is positive definite (decreasing),

2) $D^{+} U$ is nonpositive definite and $D^{+} U(t, 0)=0 \forall t \in T_{\tau}$.

Let $A S(\phi)$ be the set of MLF solving the problem of asymptotic stability of state $x=0$.

Definition 3.3. A MF $U: \quad T_{\tau} \times \mathbb{R}^{n} \rightarrow \mathbb{R}^{m \times m}$ is called a MLF of $A S(\phi)$-type on $T_{\tau} \times N$ if

1) $U$ is positive definite (decreasing),

2) $D^{+} U$ is negative definite and

$$
D^{+} U(t, 0)=0 \quad \forall t \in T_{\tau} .
$$

Let $N S(\phi)$ be the set of MF's solving the problem of instability of state $x=0$. 
Definition 3.4. A MF $U: \quad T_{\tau} \times \mathbb{R}^{n} \rightarrow \mathbb{R}^{m \times m}$ is called a Lyapunov-Chetayev MF of $N S(\phi)$-type if there exist a $t_{0} \in T$, a $\Delta>0$, such that $\bar{B}(\Delta) \subset N$ and an open set $W \subset B(\Delta)$ such that on $T_{0} \times W$

a) $\quad 0<U(t, x) \leq C$, where $C$ is an $m \times m$ real-valued matrix;

b) $\quad \phi^{\mathrm{T}} D^{+} U(t, x) \phi \geq a(v(t, x, \phi)), a$ is an element of Hahn class $K$

c) $\quad 0 \in \partial W$;

d) $\quad U=0$ on $T_{0} \times(\partial W \cap B(\Delta))$.

\section{THEOREMS OF DIRECT LYAPUNOV METHOD IN TERMS OF MATRIX-FUNCTIONS ${ }^{3}$}

The meaning of the introduced definition of MLF's is revealed in view of the following classical results of stability theory of motion.

Theorem 4.1. In order that the trivial solution of the equation (2.1) to be (uniformly) stable, it is necessary and sufficient that for (2.1) there exists a MLF $U: \quad T_{\tau} \times \mathbb{R}^{n} \rightarrow \mathbb{R}^{m \times m}$ of $S(\phi)$-type for any positive integer $m$.

Theorem 4.2. In order that the trivial solution of the equation (2.1) to be (uniformly) asymptotically stable, it is necessary and sufficient that for (2.1) there exists a MLF $U: T_{\tau} \times \mathbb{R}^{n} \rightarrow \mathbb{R}^{m \times m}$ of $A S(\phi)$-type for any positive integer $m$.

Theorem 4.3. In order that the trivial solution of the equation (2.1) be unstable it is necessary and sufficient that for (2.1) there exists a Lyapunov-Chetayev MF $U: T_{\tau} \times \mathbb{R}^{n} \rightarrow \mathbb{R}^{m \times m}$ of $N S(\phi)$-type for any positive integer $m$.

Remark 5. $\quad$ For $m=1$ Theorems $4.1-4.3$ are reduced to the classical results on stability, asymptotic stability and non-stability obtained in $([11,12,38])$. For autonomous systems results similar to Theorems 4.1 and 4.2 are given in [6], in terms of the MF (2.2) with a given vector $\phi \triangleq b\left(b_{i} \neq 0, i=1, \ldots, m\right)$ (see Remark 2$)$.

\section{METIIODS OF CONSTRUCTION OF MLFs}

We consider time-invariant nonlinear system

${ }^{3}$ The proofs to Theorem 4.1-4.3 are not yet published and are contained in a monograph by the author that will appear later. 


$$
\frac{d x}{d t}=f(x)
$$

where $x \in \mathbb{R}^{n}$ and $f \in C\left(\mathbb{R}^{n}, \mathbb{R}^{n}\right)$ is subject to physical or mathematical decomposition into $m$ interconnected subsystems

$$
\frac{d x_{i}}{d t}=g_{i}\left(x_{i}\right)+h_{i}(x) \triangleq f_{i}(x), i=1,2, \ldots, m .
$$

Here $x_{i} \in \mathbb{R}^{n_{i}}, \quad x=\left(x_{1}^{\mathrm{T}}, x_{2}^{\mathrm{T}}, \ldots, x_{m}^{\mathrm{T}}\right)^{\mathrm{T}}, f=\left(f_{1}^{\mathrm{T}}, f_{2}^{\mathrm{T}}, \ldots, f_{m}^{\mathrm{T}}\right)^{\mathrm{T}}, t \in \mathbb{R}, t_{0}=0$. The free subsystems corresponding to the system (5.2), contain the main information on dynamic properties of the system (5.2):

$$
\frac{d x_{i}}{d t}=g_{i}\left(x_{i}\right), i \in\{1, \ldots, m\}
$$

Assumption 5.1. There exist open connected neighborhoods $N_{i}$ of states $x_{i}=0$ $\forall i \in\{1, \ldots, m\}$ such that

a) motions $x_{i}\left(t, x_{i 0}\right)$ of subsystems (5.3) are continuous on

$$
\mathbb{R}_{+} \times N_{i} \text { and } x_{i}\left(0, x_{i 0}\right)=0 \quad \forall i \in\{1, \ldots, m\} ;
$$

b) motion $x\left(t, x_{0}\right)$ of system (5.1) is continuous on $\in \mathbb{R}_{+} \times N$, where $N=N_{1} \times \ldots N_{m}$ and $x\left(0, x_{0}\right)=0$.

The decomposition of system (5.1) into interconnected subsystems (5.2) is called the first level decomposition, which corresponds to the following approach of the construction of the MLF.

In matrix function (2.2) the diagonal elements $v_{i i}(x)$, are subject to the dynamical properties of the free subsystems (5.3) and the non-diagonal elements $v_{i j}\left(x_{i}, x_{j}\right)$ are constructed in response with interconnections $h_{i}(x), \forall i, j \in\{1, \ldots, m\}$. Therefore, the MLF for the system (5.2) has the form

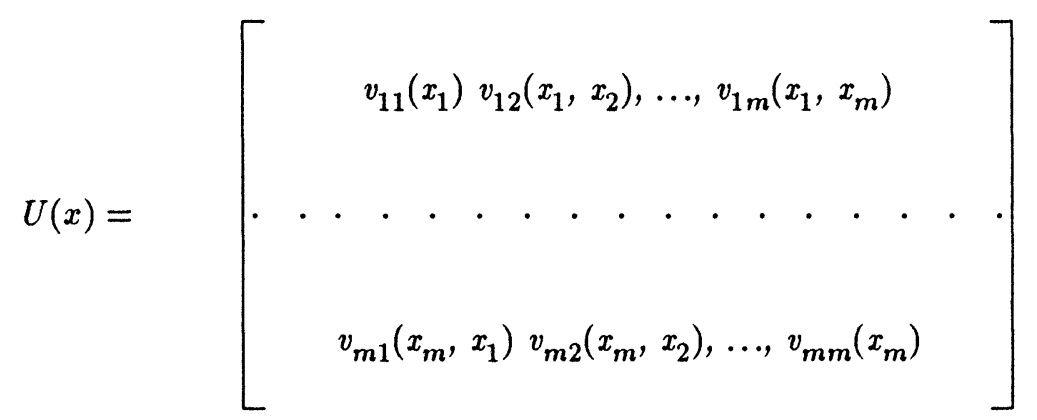

The definiteness of this MLF is defined according to Definitions 2.1 and 2.2 and Remark 2. It is possible by the construction of a vector-function to implement the interconnections $h_{i}, i=1, \ldots, m$. However, even in this case, the use of a MF improves the estimate of the region of 
asymptotic stability [cf., 36].

Example 1. (See [3]). Let $x \in \mathbb{R}^{n}$ and let system (5.1) be linear. Then, setting $x=\left(x_{1}^{\mathrm{T}}, x_{2}^{\mathrm{T}}\right)^{\mathrm{T}}$, where $x_{i} \in \mathbb{R}^{n_{i}}, n_{1}+n_{2}=n$, we have

$$
\frac{d x}{d t}=\left[\begin{array}{ll}
A_{11} & A_{12} \\
A_{21} & A_{22}
\end{array}\right] x .
$$

The elements of the matrix $U$ are taken in form

$$
v_{i i}\left(x_{i}\right)=x_{i}^{\mathrm{T}} C_{i i} x_{i} ; v_{i j}\left(x_{i}, x_{j}\right)=v_{j i}\left(x_{j}, x_{i}\right)=x_{i}^{\mathrm{T}} C_{i j} x_{j},
$$

for $i, j=1,2$. For the function

$$
v(x, \eta)=\eta^{\mathrm{T}} U(x) \eta, \eta \in \mathbb{R}^{2}
$$

we obtain the estimate

$$
v(x, \eta) \geq u^{\mathrm{T}} B^{\mathrm{T}} C B u,
$$

where $B=\operatorname{diag}\left(\eta_{1}, \eta_{2}\right), u=\left(\left\|x_{1}\right\|,\left\|x_{2}\right\|\right)^{\mathrm{T}}, C=\left(C_{i j}\right)$,

$$
\begin{aligned}
C_{i i}=\lambda_{\min } & \left(D_{i i}\right), \\
& C_{i j}=-\operatorname{sign}\left(\eta_{i}, \eta_{j}\right) \lambda \underset{\max }{1 / 2}\left(D_{i j} D_{i j}^{T}\right), i, j=1,2 .
\end{aligned}
$$

Obtaining the total derivative of the function $v$ in (5.5) by virtue of system

$$
\frac{d x_{i}}{d t}=A_{i i} x_{i}+\sum_{\substack{i=1 \\ j \neq i}}^{2} A_{i j} x_{j},
$$

it is easy to get the upper bound

$$
D v(x, \eta) \leq u^{\mathrm{T}} Q u
$$

where $Q$ is a $2 \times 2$-matrix constructed in terms of certain estimates of the derivatives of the components of the MF $U$ [cf. 2, 28]. The stability conditions for the state $\left(x_{1}^{\mathrm{T}}, x_{2}^{\mathrm{T}}\right)^{\mathrm{T}}=0$ of system (5.7) are formulated by means of the definiteness of the matrices $A$ and $Q$.

For an autonomous nonlinear large scale system (LSS) an algorithm for construction of estimate of asymptotic stability (ASD) is developed by means of a MLF (see [9]). The algorithm is as follows. Consider a class of MF's, whose $m \times m$ elements are positive definite forms. A class of scalar functions (SF) is also, constructed based on the class of MLF's as a subclass of MF's, i.e. for $U\left(x, B_{i j}\right) \in M F, B_{i j}=B_{i j}^{\mathrm{T}}>0$ the function

$$
v\left(x, \eta, B_{i j}\right)=\eta^{\mathrm{T}} U\left(x, B_{i j}\right) \eta, \eta \in \mathbb{R}_{+}^{s},
$$


is constructed, where $\left(\eta, B_{i j}\right) \in \stackrel{\circ}{\mathbb{R}}_{+}^{s} \times \mathscr{P}=\mathscr{P} S$ is a space of admissible parameters. Given a matrix-function $U\left(x, B_{i j}\right) \in M F$, the estimate of the ASD of LSS

$$
\mathscr{D}=\left\{x \in \mathbb{R}^{n}: v\left(x, \eta, B_{i j}\right)<\mu\left(\eta, B_{i j}\right)\right\}
$$

is made such that

$$
D v\left(x, \eta, B_{i j}\right)=\eta^{\mathrm{T}} D U\left(x, B_{i j}\right) \eta<0 \quad \forall(x \neq 0) \in \mathfrak{D}
$$

and

$$
\begin{aligned}
& \mu\left(\eta, B_{i j}\right)=\min _{x \in \mathcal{Z}} v\left(x, \eta, B_{i j}\right), \\
& \mathcal{J}=\left\{x \in \mathbb{R}^{n}: \operatorname{Dv}\left(x, \eta, B_{i j}\right)=0\right\} .
\end{aligned}
$$

The hypervolume of the set $\mathscr{D}$ in $\mathbb{R}^{n}$ is taken as its measure, and the problem of ASD extension is reduced to the problem of the conditional maximization.

Remark $\underline{6}$. In the MF (5.4) the diagonal elements $v_{i i}\left(x_{i}\right)$ are constructed in order to satisfy the inequalities

$$
\underline{\alpha}_{i i} \phi_{i}\left(\left\|x_{i}\right\|\right) \leq v_{i i}\left(x_{i}\right) \leq \bar{\alpha}_{i i} \psi_{i}\left(\left\|x_{i}\right\|\right), i=1,2, \ldots, m,
$$

where $\underline{\alpha}_{i i}, \bar{\alpha}_{i i}>0, \phi_{i}, \psi_{i} \in K$ are of Hahn class $K(K \mathbb{R})$, and nondiagonal elements satisfy the inequalities

$$
\underline{\alpha}_{i j} \phi_{i}\left(\left\|x_{i}\right\|\right) \phi_{j}\left(\left\|x_{j}\right\|\right) \leq v_{i j}\left(x_{i}, x_{j}\right) \leq \bar{\alpha}_{i j} \psi_{i}\left(\left\|x_{i}\right\|\right) \psi_{j}\left(\left\|x_{j}\right\|\right)
$$

where $\underline{\alpha}_{i j}, \bar{\alpha}_{i j}=$ const, $(i \neq j) \in\{1, \ldots, m\}$.

Another way of a MF construction for system (5.1) appeared in [21] where there were considered pairs $(i, j),(i \neq j) \in\{1, \ldots, m\}$ of the interconnected subsystems

$$
\begin{aligned}
& \frac{d x_{i}}{d t}=q_{i}\left(x_{i}, x_{j}\right) ; \\
& \frac{d x_{j}}{d t}=q_{i}\left(x_{j}, x_{i}\right),
\end{aligned}
$$

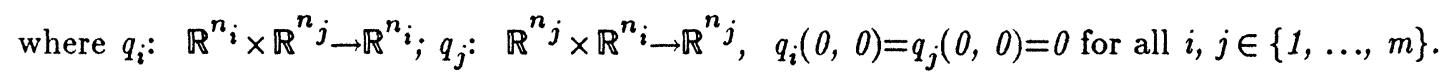

Such a decomposition is naturally referred as the second level decomposition, and the MF here is regarded as a hierarchical one $[27,9]$. The MF elements

$$
U(x)=\left(v_{i j}(\cdot)\right), i, j \in\{1, \ldots, m\}
$$

are defined as 


$$
v_{i j}=\mid \begin{gathered}
v_{i i}\left(x_{i}\right) \text { for } i=j \\
v_{i j}\left(x_{i j}\right) \text { for } i<j \\
v_{j i}\left(x_{j i}\right) \text { for } i>j,(i \neq j) \in\{1, \ldots, m\}
\end{gathered}
$$

The functions $v_{i j} \in C\left[\mathbb{R}^{n_{i}} \times \mathbb{R}^{n}, \mathbb{R}\right]$ correspond to the $(i, j)$ - pairs of (5.11). Under some assumptions on the functions $v_{i j}$ and their total Dini derivatives by virtue of systems (5.11) and interconnected system (5.1), sufficient conditions for stability of equilibrium state $x=0$ are obtained (see $[9,21])$.

\section{MATRIX-FUNCTIONS FOR SINGULARLY-PERTURBED SYSTEMS}

It is known that systems of the type

$$
\begin{gathered}
d x / d t=f(t, x, y), \\
\mu \mathrm{dy} / \mathrm{dt}=\mathrm{g}(\mathrm{t}, \mathrm{x}, \mathrm{y}, \mu),
\end{gathered}
$$

where $\left(x^{\mathrm{T}}, y^{\mathrm{T}}\right)^{\mathrm{T}} \in \mathbb{R}^{m+n}, \mu \in[0,1]$ and $t$ and $g$ are vector-functions continuous in all their arguments, are of a considerable importance for applications. This is the most general example of systems admitting the motion separations for $\mu \rightarrow 0$. In $[19,33]$, it was proposed to use the matrix-function

$$
U(t, x, y, \mu)=\left[\begin{array}{cc}
v_{11}(t, x), & v_{12}(t, x, y, \mu) \\
v_{21}(t, x, y, \mu), & v_{22}(t, y, \mu)
\end{array}\right]
$$

for solving the problem on stability of equilibrium of state $x=0$ in the equations $(6.1),(6.2)$.

We consider two concrete classes of systems $(6.1),(6.2)$ and matrix-functions corresponding to them.

CASE A. Linear singularly-perturbed system has the form

$$
\begin{gathered}
d x / d t=A_{11} x+A_{12} y, \\
\mu d y / d t=A_{21} x+A_{22} y,
\end{gathered}
$$

where $A_{11} \ldots A_{12}$ are constant matrices of the corresponding dimensions. For convenience, the elements of matrix-function (6.3) are taken in the form

$$
\begin{gathered}
v_{11}(x)=x^{\mathrm{T}} B_{1} x, v_{22}(y, \mu)=\mu y^{\mathrm{T}} B_{2} y, \\
v_{12}(\mathrm{x}, \mathrm{y}, \mu)=v_{21}(\mathrm{x}, \mathrm{y}, \mu)=\mu \mathrm{x}^{\mathrm{T}} B_{3} y,
\end{gathered}
$$


where matrices $B_{1}, B_{2}$ are symmetric positive-definite and $B_{3}$ is a constant matrix, similarly to the conditions from Example 1, for various types of stability of state $x=0$ of $(6.4),(6.5)$ were obtained by means of functions of the type of (5.5) for $\mu \in($

$\left(0, \mu^{0}\right]$ and for $\mu \rightarrow 0$, (see [34]).

\section{STABILITY OF HYBRID SYSTEMS}

The problems of hybrid system stability in sense of Lyapunov are of essential interest both in theoretical and application aspects. Recently a problem of hybrid system practical stability has also been considered (see $[20,25]$ ). The hybrid interconnected system (HIS) is a model of a really functioning system, a part of which is usually described by a (not necessarily linear) operator $L$ and the rest of which is described by a system of ordinary differential equations (ODE's). In many cases, the operator $L$ describes the object and the system of ODE's describes the regulator. In general, the operator $L$ may represent either finite-dimensional system ODE's, infinitedimensional system, delay equations, partial differential equations, Volterra integral equations or integro-differential equations. For the systems constructed by the operator $L$, are introduced the Banach space $E=\prod_{i=1} E_{i}$ is introduced, where $E_{i}$ are Banach spaces corresponding to the subsystems $\left(\sigma_{k}, g_{k}\right), k \in\{1, \ldots, m\}$ with norms $\|\cdot\|_{k}$. Here $\sigma_{k}$ is a free $k$-th subsystem, $g_{k}$ is the $k$-th interconnection function, the pair $\left(\sigma_{k}, g_{k}\right)$ determines the $k$-th interconnected subsystem $\widetilde{\sigma}_{k}$.

To be specific, we suppose that the HIS consists of isolated subsystems

$$
\sigma_{i}: \frac{d x_{i}}{d t}=X_{i}\left(t, x_{i}\right), X_{i}(t, 0)=0, i=1,2, \ldots, m
$$

where $X_{i}: T_{\tau} \times E_{i} \rightarrow E_{i}$ are connected by interconnection functions

$$
g_{i}: g_{i}=g_{i}\left(t, x_{1}, \ldots, x_{m}\right), i \in\{1, \ldots, m\}
$$

and such that $g_{i}: T_{\tau} \times E_{1} \times \ldots \times E_{m} \rightarrow E_{i}$.

The fixed interacting $k$-th subsystem is described by the pair

$$
\left(\sigma_{k}, g_{k}\right): \frac{d x_{k}}{d t}=X_{k}\left(t, x_{k}\right)+g_{k}\left(t, x_{1}, \ldots, x_{m}\right),
$$

where $x_{k} \in E_{k}$. We introduce some assumptions.

Assumption 7.1. There exist connected neighborhoods $N_{i} \subseteq E_{i}$ of states $x_{i}=0$ $\forall i=1,2, \ldots, m$ such that

a) the motions $x_{i}\left(t, t_{0}, x_{i}^{0}\right)$ of subsystems (7.1) are continuous on $\left(t, x_{i 0}\right) \in T_{\tau} \times N_{i}$ where $x\left(0 ; t_{0}, x_{i 0}\right)=0, i=1,2, \ldots, m$; 
b) the motion $x\left(t ; t_{0}, x_{0}\right)$ of HIS is continuous on $\left(t, x_{0}\right) \in \tau_{\tau} \times N$, where $N=N_{1} \times N_{2} \times \ldots \times N_{m}$ and $x\left(0 ; t_{0}, x_{0}\right)=0$.

Let $U \in C\left[T_{\tau} \times E, \mathbb{R}^{m \times m}\right]$ be a MF for system (7.3). Like the functions $U_{0}(t, x)$ for the system of ordinary differential equations by (2.3), Theorems $4.1-4.3$ are formulated for hybrid system (7.1) - (7.2).

Further we consider a vector approach in terms of matrix-function. Define a vector-function as

$$
L(t, x)=A U(t, x) \phi
$$

where $A$ is a constant $m \times m$ matrix, $\phi \in \mathbb{R}_{+}^{m}$ or $\phi \in C\left[\mathbb{R}^{n}, \mathbb{R}_{+}\right],(\phi(0)=0)$. For $(t, x) \in T_{\tau} \times E$ we introduce the Dini derivative of vector-function $L(t, x)$ by the formula

$$
D^{+} L(t, x)=\lim _{h \rightarrow 0^{+}} \sup \frac{1}{h}[L(t+h, x+h X(t, x))-L(t, x)]
$$

It should be noted that if $\phi \in \mathbb{R}_{+}^{m}$, then

$$
D^{+} L(t, x)=A D^{+} U(t, x) \phi
$$

where $D^{+} U(t, x)$ is defined similarly to $(7.5)$.

Lemma 7.1. Let there exist

1) a matrix-function $U(t, x) \in C\left[\mathbb{R}_{+} \times E, \mathbb{R}^{m \times m}\right]$, such that $U(t, x)$ is locally Lipschitz in $x$ for $(t, x) \in \mathbb{R}_{+} \times E$;

2) a constant $m \times m$ matrix $A$, a vector $\phi \in \mathbb{R}_{+}^{m}$ and a vector $\eta \in \mathbb{R}_{+}$such that

$$
\eta^{\mathrm{T}} L(t, x) \geq a(\|x\|)
$$

where $a$ is from Hahn class $K$.

3) a vector-function $G \in C\left[\mathbb{R}_{+} \times \mathbb{R}_{+}^{m}, \mathbb{R}_{+}^{m}\right]$, such that $G(t, u)$ is quasimonotone nondecreasing on $u$ for every $t \in \mathbb{R}_{+}$and such that the estimate

$$
D^{+} L(t, x) \leq G(t, L(t, x))
$$

holds.

4) a maximal solution $r\left(t, t_{0}, u_{0}\right)$ of the system

$$
d u / d t=G(t, u), u\left(t_{0}\right)=u_{0} \geq 0
$$

on interval $\left[t_{0}, \infty\right)$.

Then the inequality

$$
L\left(t, x\left(t, t_{0}, x_{0}\right)\right) \leq r\left(t, t_{0}, u_{0}\right),
$$

holds with respect to the solutions $x\left(t, t_{0}, x_{0}\right)=\left(x_{1}^{\mathrm{T}}\left(t, t_{0}, x_{10}\right), \ldots, x_{m}^{\mathrm{T}}\left(t_{1}, t_{0}, x_{m}\right)\right)^{\mathrm{T}}$ of hybrid system (7.3) as long as 


$$
L\left(t_{0}, x_{0}\right) \leq u_{0}
$$

The lemma is proved in a standard way by the comparison method (see [12] and [13]).

Lyapunov stability of hybrid system (7.3) will be investigated with respect to a measure $\rho=\|x\|$, where $\|x\|=\left\|x_{1}\right\|_{1}+\ldots+\left\|x_{m}\right\|_{m}$. For such a choice of measure for system (7.3) defined in spaces $L_{p}$, norms are defined by the formula

$$
\left.\left\|x_{s}\right\|_{L_{p}}=\int_{a}^{b}\left\|x_{s}(\tau)\right\|^{p} d \tau\right)^{1 / p}, p \in[1, \infty),
$$

$1 \leq s<m$. To formulate stability definitions for (7.3) we suppose that

1) $\quad X_{k}\left(t, x_{k}\right) \equiv 0$ for $x_{k} \equiv 0$ and $g_{k}(t, 0, \ldots, 0) \equiv 0, k=1,2, \ldots, m$;

2) $\quad G(t, u) \equiv 0$ for $u \equiv 0$ for all $t \in \mathbb{R}_{+}$.

Definition 7.1. The equilibrium state $x=0$ of hybrid system (7.3) is called conditionally equi-stable, if for every $\epsilon>0$ and $t_{0} \in \mathbb{R}_{+}$there exists $\delta=\delta\left(t_{0}, \epsilon\right)>0$ such that $\left\|x_{0}\right\|<\delta$ implies $\left\|x\left(t, t_{0}, x_{0}\right)\right\|<\epsilon$ for all $t \geq t_{0}$.

Definition 7.2. The trivial solution $u=0$ of comparison system (7.9) is called conditionally equi-stable, if for every $\epsilon^{*}>0$ and $t_{0} \in \mathbb{R}_{+}$there exists $\delta^{*}=\delta^{*}\left(t_{0}, \epsilon^{*}\right)$ such that $u_{0} \in H^{+} \subset \mathbb{R}_{+}^{m}$ and $\sum_{i=1}^{m} u_{i 0}<\delta$ imply the inequality $\sum_{i=1}^{m} u_{i}\left(t, t_{0}, u_{0}\right)<\epsilon^{*}$ for all $t \geq t_{0}$.

Theorem 7.1. $\quad$ Let

1) conditions 1 - 3 of Lemma 7.1 be satisfied

2) zero solution of (7.8) be

a) conditionally equi-stable;

b) uniformly asymptotically stable;

Then the equilibrium of state $x=0$ of (7.3) is

a) conditionally equi-stable;

b) uniformly asymptotically stable, if for some function $b$ of class $K$ $\eta^{\mathrm{T}} L(t, x) \leq b(\|x\|)$.

The theorem can be proved in a standard way applied for the proofs of stability theorems in the comparison method for equations in Banach spaces.

\section{STABILITY ANALYSIS OF TWO-COMPONENT SYSTEM}

We consider a system consisting of two subsystems, one of which is modeled by an ordinary 
differential equation and the other one by a partial differential equation (see [1]). Let the interval $T_{0}=\left[t_{0},+\infty\right)$ and a bounded domain $\Omega$ be given in space $\mathbb{R}^{k}$. Consider the problem

$$
\begin{aligned}
& d x / d t=X(t, x(t))+\mu g_{1}(t, z, x(t), w(t, z)), x\left(t_{0}\right)=x_{0}, \\
& d w / d t=L\left(t, z, \frac{\partial}{\partial z}\right) w+\mu g_{2}(t, z, x(t), w(t, z)), \\
& w\left(t_{0}, z\right)=w^{0}(z),\left.M\left(t, z, \frac{\partial}{\partial z}\right) \phi\right|_{\partial \Omega}=w^{1}(t, x), s \in \partial \Omega .
\end{aligned}
$$

Here $\mu>0$ is a small positive parameter, $x: \tau_{0} \rightarrow H, H \subseteq \mathbb{R}^{n}$;

$$
w: \quad T_{0} \times \Omega \rightarrow \mathrm{Q}, \mathrm{Q} \subseteq \mathbb{R}^{m} ; X: T_{0} \times H \rightarrow \mathbb{R}^{n} ; g_{1}: T_{0} \times \Omega \times H \times Q \rightarrow \mathbb{R}^{n} ;
$$

$L: \quad B_{1} \rightarrow B_{2}, M: \quad B_{1} \rightarrow B_{2}, M: \quad B_{1} \rightarrow B_{3}, w^{0} \in B_{4} ; g_{2}: T_{0} \times \Omega \times H \times Q \rightarrow \mathbb{R}^{m}$.

Because of the presence of the small parameter $\mu$ in the interconnection functions of subsystems

$$
\begin{gathered}
d x / d t=X(t, x(t)), x\left(t_{0}\right)=x_{0} \\
d w / d t=L\left(t, z, \frac{\partial}{\partial z}\right) w, w\left(t_{0}, z\right)=w^{0}(z) ; \\
\left.M\left(t, z, \frac{\partial}{\partial z}\right) \phi\right|_{\partial \Omega}=w^{1}(t, s), s \in \partial \Omega
\end{gathered}
$$

the system (8.1) - (8.2) is a weakly connected hybrid systems (see [30]).

\section{Assumption 1. There exist}

1) functions $v_{11} \in C\left[T_{0} \times N_{x}, \mathbb{R}_{+}\right]$, such that $v_{11}(t, x)$ is locally Lipschitz in $x$, $v_{22} \in C\left[T_{0} \times N_{w}, \mathbb{R}_{+}\right]$, such that $v_{22}(t, x)$ is locally Lipschitz on $w$, and $N_{x} \subseteq H, N_{w} \subseteq B_{1}$ are open connected neighborhoods of points $x=0$ and $w=0$ respectively;

2) functions $\phi_{i}(\|x\|)$ and $\psi_{i}(\|w\|)$ of Hahn class $K, i=1,2$;

3) functions $v_{12}(t, x, w)=v_{21}(t, x, w), v_{12} \in C\left[T_{0} \times N_{x} \times N_{w}, \mathbb{R}\right]$, such that $v_{12}(t, x, w)$ and $v_{21}(t, x, w)$ are locally Lipschitz in $x$ and $w$;

4) positive constants $\underline{\alpha}_{i i}$ and $\bar{\alpha}{ }_{i i}, i=1,2$, and arbitrary constants $\underline{\alpha}_{12}$ and $\bar{\alpha}_{12}$ for which the estimates
a) $\quad \underline{\alpha}_{11} \phi_{1}^{2}(\|x\|) \leq v_{11}(t, x) \leq \bar{\alpha}_{11} \phi_{2}^{2}(\|x\|)$;
b) $\quad \underline{\alpha}_{22} \psi_{1}^{2}(\|w\|) \leq v_{22}(t, w) \leq \bar{\alpha}_{22} \psi_{2}^{2}(\|w\|)$;
c) $\quad \underline{\alpha}_{12} \phi_{1}(\|x\|) \psi_{1}\left(\|w\| \leq v_{12}(t, x, w) \leq \bar{\alpha}_{12} \phi_{2}(\|x\|) \psi_{2}(\|w\|)\right.$.

hold.

Assumption 1 implies the following lemma. 


$$
\begin{gathered}
A_{1}=\left[\begin{array}{cc}
\underline{\alpha}_{11} & \underline{\alpha}_{12} \\
\underline{\alpha}_{21} & \underline{\alpha}_{22}
\end{array}\right] \quad A_{2}=\left[\begin{array}{cc}
\bar{\alpha}_{11} & \bar{\alpha}_{12} \\
\bar{\alpha}_{21} & \bar{\alpha}_{22}
\end{array}\right] \\
\underline{\alpha}_{12}=\underline{\alpha}_{21} ; \quad \bar{\alpha}_{21}=\bar{\alpha}_{12}
\end{gathered}
$$

are positive-definite, the function

$$
v(t, x, w)=\phi^{\mathrm{T}} U(t, x, w) \phi
$$

(where $\left.\phi^{\mathrm{T}}=\left(\eta_{1}, \eta_{2}\right)-\eta_{1}, \eta_{2}>0\right)$ is positive-definite and decreasing.

\section{Assumption 2. There exist}

1) functions $v_{i j}(t, \cdot)$ mentioned in Assumption 1 ;

2) constants $\beta_{i k}, i=1,2 ; k=1,2, \ldots, 8$, functions $\zeta_{1}=\zeta_{1}(\|x\|)$ and $\zeta_{2}(\|w\|)$, $\zeta_{1}, \zeta_{2}$ are of Hahn class $K$ and estimates
a) $\quad D_{t}^{+} v_{11}+\left(D_{x}^{+} v_{11}\right)^{\mathrm{T}} X \leq 0$
b) $\quad\left(D_{x}^{+} v_{11}\right)^{\mathrm{T}} g_{1} \leq \beta_{22} \zeta_{1}^{2}+\beta_{13} \zeta_{1} \zeta_{2}$;
c) $\quad D_{t}^{+} v_{22}+\left(D_{w}^{+} v_{22}\right)^{\mathrm{T}} L \leq 0$;
d) $\quad\left(D_{w}^{+} v_{22}\right)^{\mathrm{T}} g_{2} \leq \beta_{22} \zeta_{2}^{2}+\beta_{23} \zeta_{1} \zeta_{2}$;
e) $\quad D_{t}^{+} v_{12}+\left(D_{x}^{+} v_{12}\right)^{\mathrm{T}} X \leq \beta_{14} \zeta_{1}^{2}+\beta_{15} \zeta_{1} \zeta_{2}$;
f) $\quad\left(D_{w}^{+} v_{12}\right)^{\mathrm{T}} L \leq \beta_{24} \zeta_{1}^{2}+\beta_{25} \zeta_{1} \zeta_{2}$;
g) $\quad\left(D_{x}^{+} v_{12}\right)^{\mathrm{T}} g_{1} \leq \beta_{16} \zeta_{1}^{2}+\beta_{17} \zeta_{1} \zeta_{2}+\beta_{18} \zeta_{2}^{2}$;
h) $\quad\left(D_{w}^{+} v_{12}\right)^{\mathrm{T}} g_{2} \leq \beta_{26} \zeta_{1}^{6}+\beta_{23} \zeta_{1} \zeta_{2}+\beta_{28} \zeta_{2}^{2}$

hold.

Consider matrix $C(\mu)$ in form

$$
C(\mu)=\left[\begin{array}{ll}
C_{11} & C_{12} \\
C_{21} & C_{22}
\end{array}\right], C_{12}=C_{21}
$$

with the elements 


$$
\begin{aligned}
& C_{11}=\eta_{1}^{2} \mu \beta_{12}+2 \eta_{1} \eta_{2}\left(\beta_{14}+\mu \beta_{16}+\mu \beta_{26}\right) \\
& C_{22}=\eta_{2}^{2} \mu \beta_{22}+2 \eta_{1} \eta_{2}\left(\beta_{24}+\mu \beta_{18}+\mu \beta_{28}\right) \\
& C_{12}=\frac{1}{2}\left(\eta_{1}^{2} \mu \beta_{13}+\eta_{2}^{2} \mu \beta_{23}\right)+\eta_{1} \eta_{2}\left(\beta_{15}+\beta_{25}+\mu \beta_{17}+\mu \beta_{27}\right) .
\end{aligned}
$$

Introduce designations $a, p, q ; \mu_{1}, \mu_{2}, \mu_{3}, \mu_{4}$ by formulas

$$
\begin{aligned}
& a=\eta_{1} \eta_{2}\left[\eta_{1} \beta_{12}+2 \eta_{1}\left(\beta_{16}+\beta_{26}\right)\right]\left[\eta_{2} \beta_{22}+2 \eta_{1}\left(\beta_{13}+\beta_{28}\right)\right]- \\
& -\left[\frac{1}{2}\left(\eta_{1}^{2} \beta_{13}+\eta_{2}^{2} \beta_{23}\right)+\eta_{1} \eta_{2}\left(\beta_{17}+\beta_{27}\right)\right]^{2} \\
& p=\eta_{1} \eta_{2}\left\{\left[\eta_{1} \beta_{24}\left(\eta_{1} \beta_{12}+2 \eta_{1} \beta_{16}+2 \eta_{2} \beta_{26}\right)+\eta_{2} \beta_{14}\left(\eta_{2} \beta_{22}+2 \eta_{1} \beta_{18}+\right.\right.\right. \\
& \left.\left.\left.+2 \eta_{1} \beta_{28}\right)\right]-\left(\beta_{15}+\beta_{25}\right)\left[\frac{1}{2}\left(\eta_{1}^{2} \beta_{13}+\eta_{2}^{2} \beta_{23}\right)+\eta_{1} \eta_{2}\left(\beta_{17}+\beta_{27}\right)\right]\right\} ; \\
& \left.q=\eta_{1}^{2} \eta_{2}^{2}\left[4 \beta_{14} \beta_{24}-\left(\beta_{15}+\beta_{25}\right)^{2}\right)\right) \text {; } \\
& \mu_{1}=-2 \eta_{1} \beta_{14} / \eta_{1}\left(\beta_{12}+2 \eta_{2}\left(\beta_{16}+\beta_{26}\right)\right) \text {; } \\
& \mu_{2}=-2 \eta_{1} \beta_{24} / \eta_{2} \beta_{22}+2 \eta_{1}\left(\beta_{18}+\beta_{28}\right) \\
& \mu_{3}=\left(p+\sqrt{p^{2}-4 a q}\right)(-2 a)^{-1} \text {; } \\
& \mu_{4}=\left(-p-\sqrt{p^{2}-4 a q}\right)(2 a)^{-1} \text {. }
\end{aligned}
$$

Further consider a value of parameter $\mu$, whose boundary value is determined by the conditions

$\mathrm{B}_{0} . \quad$ If $a>0, p>0$, then $\mu_{0}=\min \left(\mu_{1}, \mu_{2}\right)$.

$\mathrm{B}_{1}$. If $a>0$ and $p$ is arbitrary, then $\mu_{0}=\min \left(\mu_{1}, \mu_{2}, \mu_{3}\right)$.

$\mathrm{B}_{2}$. If $a>0$ and $p>0$, then $\mu_{0}=\min \left(\mu_{1}, \mu_{2}, \mu_{4}\right)$.

The validity of the following lemma is easily verified.

Lemma 8.2. If all conditions in Assumption 2 are satisfied and the inequalities

a) $\quad \eta_{2} \beta_{14}<0$;

b) $\quad \eta_{1} \beta_{12}+2 \eta_{2}\left(\beta_{16}+\beta_{26}\right)>0$;

c) $\quad \eta_{1} \beta_{24}<0$;

d) $\quad \eta_{2} \beta_{22}+2 \eta_{1}\left(\beta_{18}+\beta_{28}\right)>0$;

e) $\quad 4 \beta_{14}-\left(\beta_{15}+\beta_{25}\right)^{2}>0$,

hold true, then the matrix $C(\mu)$ is negative-definite for $\mu \in] 0, \mu_{0}\left[\right.$ and $\mu \rightarrow 0$, where $\mu_{0}$ is defined by one of the conditions $\mathrm{B}_{0}-\mathrm{B}_{2}$.

In view of condition 2, Assumption 2, we have for function (8.5) the following estimate of Dini derivative

$$
D^{+} v(t, x, w) \leq u^{\mathrm{T}} C(\mu) u
$$

where $\left.u^{\mathrm{T}}=\left(\zeta_{1}(\|x\|), \zeta_{2}(\|w\|)\right), \mu \in\right] 0, \mu_{0}[$.

Theorem 8.1. If two-component system (8.1) - (8.2) is such that all conditions of 
Lemmas 8.1 and 8.2 are satisfied, then its equilibrium state $x=0, w=0$ is uniformly asymptotically stable for $\mu \in] 0, \mu_{0}[$ and for $\mu \rightarrow 0$.

The proof of the theorem follows from the conditions satisfied by the function (8.5) and its Dini derivative (8.7).

Remark 8.7. In consequence of conditions 2 a) and 2 c) of Assumption 2, the hybrid system (8.1) - (8.2) consists of (nonasymptotically) stable subsystems and uniform asymptotic stability of equilibrium state $x=0, w=0$ is achieved due to the interconnection functions stabilizing effect.

\section{REFERENCES}

[1] Bartyshev, A.V., The Application of Vector Lyapunov Functions in the Investigation of Two-Component Systems, Vector Lyapunov function and their construction. Nauka, Novosibirsk, 1980, 237-257.

[2] Djordjević, M.Z., Stability Analysis of Interconnected Systems with Possible Unstable Subsystems. Systems and Control Letters, 3 (1983), 165-169.

[3] Djordjević, M.Z., Stability Analysis of Large Scale Systems Whose Subsystems May be Unstable. Large Scale Systems, 5 (1983), 255-262.

[4] Djordjević, M.Z., Stability Analysis of Nonlinear System by the Matrix Lyapunov Method. Proc. IMACS-IFAC Symposium, June 3-6, 1986, IDN, ViWeneuve d'Ascq. France, 209-212.

[5] Grujić, Lj. T., Novel Development of Lyapunov Stability of Motion. Int. Journal of Control, 22(1975), 529-549.

[6] Grujić, Lj. T., On Large-Scale Systems Stability. Proc. 12th World Congress IMACS, 1(1988), 224-229.

[7] Grujić, Lj. T., Martynyuk, A.A., Ribbens-Pavella, M., Large-Scale System Stability Under Structural and Singular Perturbations. Berlin, Springer-Verlag, 1987.

[8] Grujić, Lj. T., Shaaban, H., On Transient Stability Analysis of Large Scale Power Systems. Proc. 12th World Congress IMACS, 1(1988), 283-288.

[9] Krapivny, Yu. N., Ways of Matrix Lyapunov Functions Construction and Estimation of Asymptotic Stability Domain of Large Scale Systems. Thesis of doctoral dissertation, Kiev, USSR, 1988.

[10] Krapivny, Yu. N., Martynyuk, A.A., Nonlinear Systems Comparison and Large Scale System Stability. Prikl. Mech., 25(1987), 75-80.

[11] Krassovsky, N.N., Some Problems of Motion Stability Theory. Moscow, GIFML, 1959. 
Lakshmikantham, V., Leela, S., Differential and Integral Inequalities. New York, Academic Press, I, II (1969).

Lakshmikantham V., Leela, S., Martynyuk, A.A., Stability Analysis of Nonlinear Systems. New York, Marcel Dekker, Inc., (1989). 5 Vol., Moscow, USSR, Acad. of Sci., Vol. 2, 1956.

Martynyuk, A.A., The Lyapunov Matrix Function. Nonlinear Analysis: Theory, Methods, and Applications, 8 (1984), 1223-1226.

Martynyuk, A.A., Matrix Lyapunov Function and Stability of Hybrid Systems. Prikl. Mech., 23(1985), No. 4, 89-96.

Marynyuk, A.A., Uniform Asymptotic Stability of Singularly Perturbed System in Terms of Matrix Lyapunov Function. Dokl. AN SSSR, 287(1986), 786-789.

Martynyuk, A.A., On Practical Stability of Hybrid Systems. Prikl. Mech., 25(1989), No. 2, 101-107.

Martynyuk, A.A., Hierachical Matrix Lyapunov Function and Stability Order under Structural Perturbations. Dokl. AN SSSR, 305(1989), No. 1, 41-44.

Martynyuk, A.A., On Stability of Stochastic Singularly Perturbed Systems. Dokl. AN SSSR, Ser. A(1989), No. 1, 55-57.

Martynyuk, A.A., On the Application of Matrix Lyapunov Functions in Motion Investigation of Lumped and Distributed Parametr Systems. Teor. Mehanika i Primenjena, 1989, No. 14, 73-83.

Martynyuk, A.A., The Development of Direct Lyapunov Method for SingularlyPerturbed System on the Basis of Matrix Function. Proc. ICNO-XII, Budapest, 1987, 149-156.

Martynyuk, A.A., Practical Stability Conditions for Hybrid Systems. Proc. 12th World Congress IMACS, 1(1988), 344-347.

Martynyuk, A.A., On Matrix Lyapunov Function for Stochastic Dynamical Systems. Proc. 12th World Congress IMACS, 1(1988), 216-219.

Martynyuk, A.A., Hierarchical Matrix Lyapunov Function, Differential and Integral Equations, 2(1989), 411-417.

Martynyuk, A.A., On Application of the Lyapunov Matrix Functions in the Theory of Stability. Nonlinear Analysis: Theory, Methods and Applications, $9(1985), 1495-1501$. 
Systems. Nonlinear Analysis: Theory, Methods and Applications, 10(1986), 1449-1457.

$[30]$

Martynyuk, A.A., Lyapunov Matrix Function and Stability Theory. Proc. IMACS-IF AC Symp. IDN, 1986, Villeneuve d'Ascq. France, 261-265.

Martynyuk, A.A., Uniform Asymptotic Stability of a Singularly Perturbed Systems Via the Lyapunov Matrix Function. Nonlinear Analysis: Theory, Methods and Applications, 11(1987), 1-4. Martynyuk, A.A., Gutowsky, R., Integral Inequalities and Stability of Motion. Kiev, USSR, Naukova Dumka, 1979.

Martynyuk, A.A., Milodzhanov, V.G., General Problem on Stability of Singularly-Perturbed Large Scale System and Method of Matrix Lyapunov Function. Preprint 86.75. Institute of Math. of Ukr. Ac. of Sci., Kiev, 1986.

Martynyuk, A.A., Milodzhanov, V.G., Stability Investigation ofAutonomony Singularly-Perturbed Systems in Terms of Matrix Lyapunov Function. Diff. Eqs., 24(1988), No. 3, 416-424.

Martynyuk, A.A., Shegai, V., To Stability Theory of Autonomony Systems. Prikl. Mech., 22(1986), No. 4, 97-102.

Martynyuk, A.A., Shegai, V., Stability Investigation of Large-Scale Systems in Terms of Matrix Lyapunov Functions. Prikl. Mech., 22(1986), No. 6, 106-113.

Michel, A.N., Miller, R.K., Qualitative Analysis of Large-Scale Dynamical Systems. New York, Academic Press, 1977.

Yoshikawa, T., Stability Theory by Lyapunov's Second Method. Tokyo, Math. Soc., Japan, 1966. 


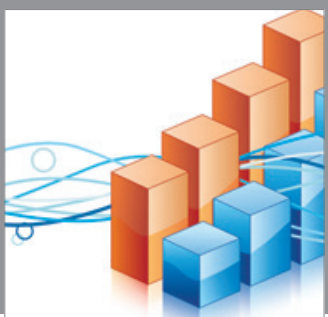

Advances in

Operations Research

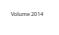

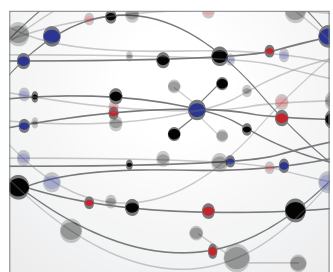

\section{The Scientific} World Journal
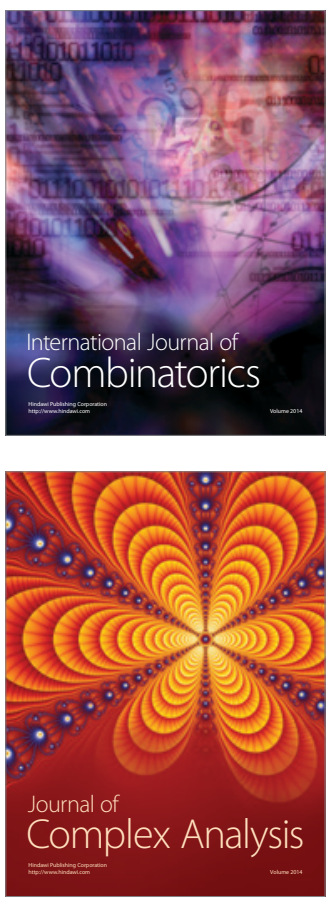

International Journal of

Mathematics and

Mathematical

Sciences
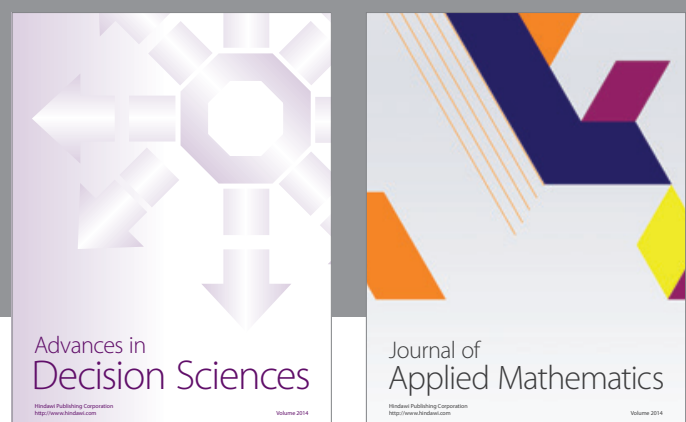

Journal of

Applied Mathematics
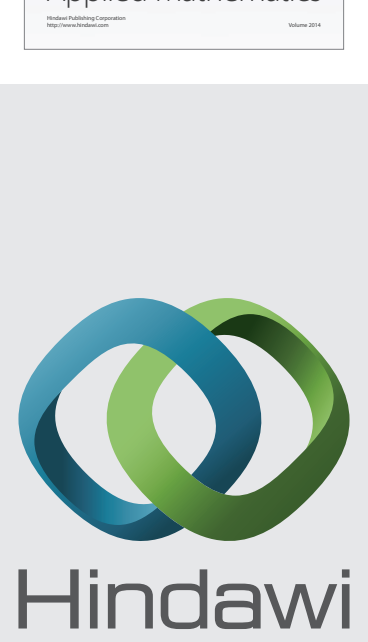

Submit your manuscripts at http://www.hindawi.com
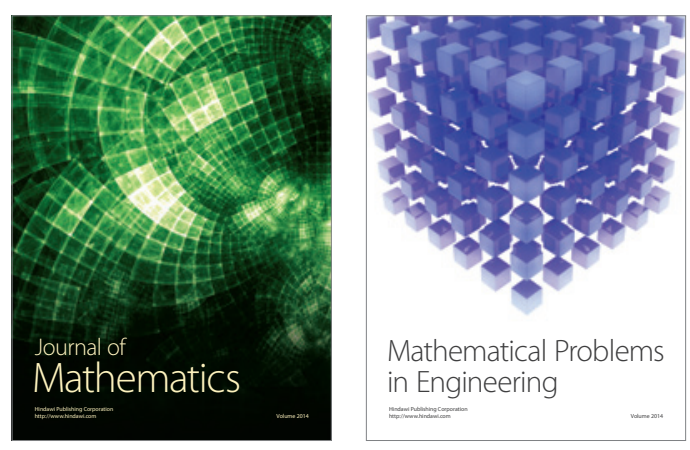

Mathematical Problems in Engineering
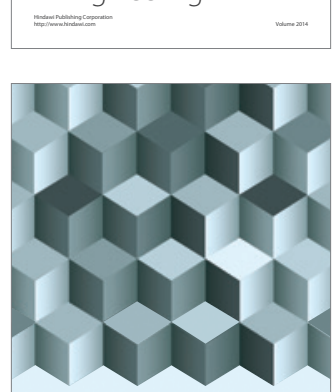

Journal of

Function Spaces
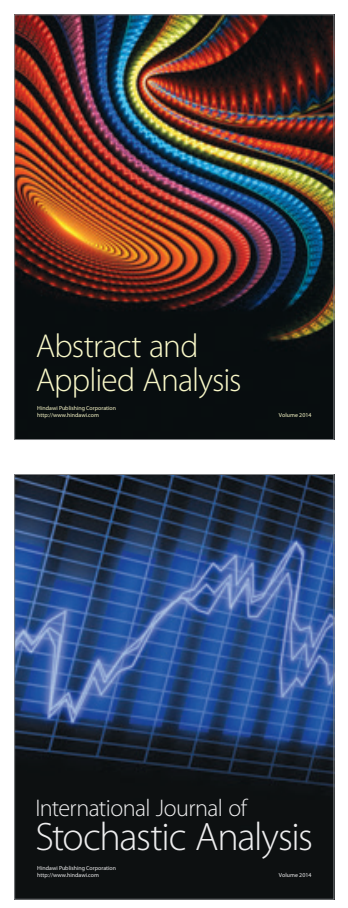

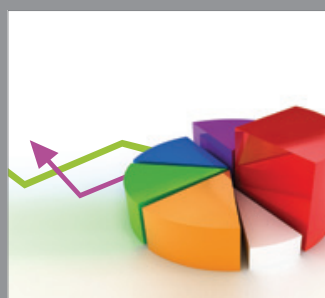

ournal of

Probability and Statistics

Promensencen
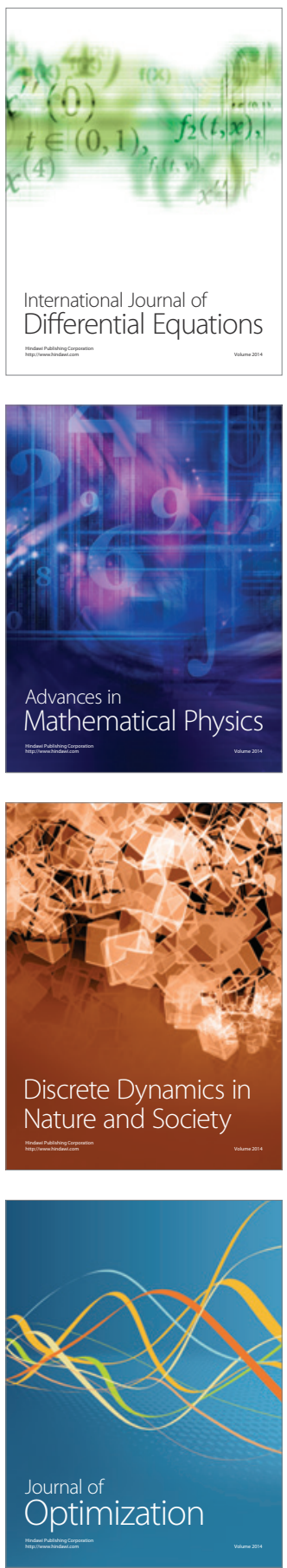